\title{
Relationships between longitudinal neutrophil to lymphocyte ratios, body weight changes, and overall survival in patients with non-small cell lung cancer
}

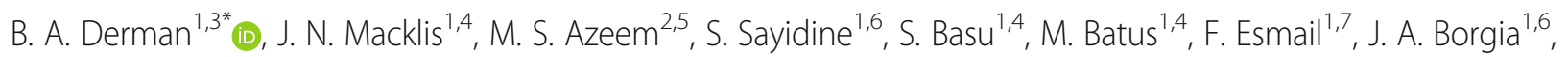
P. Bonomi $i^{1,4}$ and M. J. Fidler ${ }^{1,4}$

\begin{abstract}
Background: There is emerging evidence showing a significant relationship between overall survival (OS) in non-small cell lung cancer NSCLC patients and weight change during chemotherapy or chemoradiation. A high neutrophil/lymphocyte ratio (NLR) at baseline and at follow-up is associated with shorter survival in cancer patients and may be a surrogate for ongoing inflammation, implicated in cancer cachexia and tumor progression. The objective of this study is to explore potential relationships between OS, serial weights, and serial NLRs in advanced NSCLC patients receiving chemotherapy.

Methods: One hundred thirty-nine patients with chemotherapy-naïve NSCLC, predominantly with stage III/IV disease, were treated with first-line platinum doublets from June, 2011 to August, 2012. NLR, tumor response, and body weight were recorded at baseline, 6 , and 12 weeks from initiation of therapy and correlated with OS. The association between NLR and OS was assessed using Cox PH (proportional hazards) analysis, the association between NLR and weight change was assessed using a simple regression analysis, and the association between NLR and tumor response was assessed using the Fisher's exact test.

Results: One hundred thirty-nine patients with median age 68 , PS 0-1/2 $=83 / 17 \%$, male/female $=58 \% / 42 \%$. Median NLR at baseline was 3.6 (range 0.1898 to 30.910), at 6 weeks 3.11 (range 0.2703 to 42.11), and at 12 weeks 3.52 (range 0.2147 to 42.93). A Higher NLR at baseline, 6 , and 12 weeks was associated with decreased OS (baseline: HR 1.06, $p<0.001 ; 6$ weeks: HR 1.07, $p=0.001 ; 12$ weeks: HR 1.05, $p<0.001$ ), and longitudinal NLR, as a time-dependent covariate, was also associated with decreased OS ( $H R=1.06, p<0.001)$. Baseline weight and NLR were inversely related (cor $=-0.267, p=0.001$ ), and weight change and NLR were inversely related at 12 weeks (cor $=-0.371, p<0.001$ ). Longitudinal measurements of weight and NLR were also negatively associated (slope $=-0.06, p<0.001$ ). Using a cutoff of NLR $>5$, there was a significant association between progressive disease and NLR $>5$ at 6 weeks $(p=0.02)$ and 12 weeks $(p=0.03)$.

Conclusions: High baseline and progressive increases in NLRs are associated with progressive disease, inferior OS and weight loss in NSCLC patients. In addition to having prognostic significance, these observations suggest that studying molecular mediators of cachexia/inflammation and their relationships to tumor progression may identify new therapeutic targets in the large subset of NSCLC patients who have cancer cachexia.
\end{abstract}

Keywords: NSCLC, Weight gain, Neutrophil to lymphocyte ratio, Cancer cachexia

* Correspondence: Ben_Derman@rush.edu

${ }^{1}$ Rush University Medical Center, Chicago, IL, USA

${ }^{3}$ Department of Internal Medicine, 1717 W Congress Parkway, 1025 Kellogg,

Chicago, IL 606012, USA

Full list of author information is available at the end of the article 


\section{Background}

Cancer cachexia represents a multifactorial spectrum of disease that has been defined by international consensus as "an ongoing loss of skeletal muscle mass (with or without loss of fat mass) that cannot be fully reversed by conventional nutritional support and leads to progressive functional impairment" [1]. Prevalence rates of cachexia differ by malignancy type, with approximately $60 \%$ of NSCLC patients experiencing it [2]. Cancer cachexia may be a result of both reduced nutritional intake and increased resting energy expenditure [3]. More recent evidence suggests that there are several other factors that contribute to cancer cachexia, which includes increased insulin resistance, hypogonadism, adrenergic activation, and activation of proinflammatory responses [4]. Identifying and combating this phenomenon has taken on great importance in light of studies showing that weight change in advanced NSCLC patients receiving concurrent chemoradiation or chemotherapy alone is inversely associated with overall survival (OS) [5-7]. Similarly, loss of muscle mass (regardless of BMI) is also associated with worse functional status and OS [8].

The NLR is the ratio between the neutrophil and lymphocyte counts; what constitutes an elevated value varies between $>3.5$ and $>5$. Several studies have already shown that an increased baseline NLR is associated with poor clinical outcomes for several types of cancers, including NSCLC [9-13]. There is relatively little information regarding longitudinal NLRs, which some have posited may be even more predictive of individual patient outcomes [14] and may be a dynamic indicator of tumor status, cachexia, and ongoing inflammation. Our objective was to investigate potential relationships between longitudinal NLRs, body weights, and overall survival.

\section{Methods}

One hundred thirty-nine patients with NSCLC who were treated with first-line platinum doublets from June, 2011 to August, 2012 were reviewed for this study; none of the patients received prior therapy with an EGFR tyrosine kinase inhibitor. 127 patients had stage III or stage IV NSCLC, and 12 patients had either stage I or stage II disease and received adjuvant chemotherapy following surgical resection. This study was approved by the institutional review board, and compliant with the Helsinki declaration. NLR and body weight were recorded at baseline, 6 , and 12 weeks from initiation of therapy and correlated with OS. All patients had blood drawn in outpatient clinic on the day of evaluation and treatment for advanced NSCLC. None had active infections at the time of these visits. Neutrophil and lymphocyte counts were measured on the Sysmex XN-9000 Hematology Analyzer, Sysmex Corporation, Kobe, Japan. Albumin was measured on the Architect Clinical Chemistry Analyzer C16000, Abbott Diagnostics, Santa Clara, California. The association of OS at NLR at baseline, 6 weeks, and 12 weeks was assessed using the Kaplan-Meier method, the logrank test, and the Cox proportional hazards $(\mathrm{PH})$ analysis. The effect of serial NLR measured longitudinally on OS was assessed using a time-dependent covariate Cox PH analysis. The longitudinal association between weight and NLR was measured serially at baseline, 6 and 12 weeks was assessed using a mixed effects longitudinal analysis. The association between NLR and tumor response was assessed using a Fisher's exact test. Tumor response rates were calculated for the patients with measurable disease according to the RECIST (Response Evaluation Criteria in Solid Tumors) 1.1 guidelines. The ALI index was defined as the BMI*Albumin/NLR, and ALI was calculated at baseline, 6 and 12 weeks from initiation of therapy and correlated with OS; 96, 93, and 84 patients had ALI scores available at baseline, 6 and 12 weeks respectively.

\section{Results}

Of 139 patients evaluated, the median age was 68 years, and $58 \%$ were male. $83 \%$ had a performance score of 0 1 and $17 \%$ had a performance score of 2 (Table 1).

The median NLR at baseline (Table 2) was 3.6 (range 0.1898 to 30.910 ), at 6 weeks 3.11 (range 0.2703 to 42.11), and at 12 weeks 3.52 (range 0.2147 to 42.93 ). Higher NLR at baseline, 6 and 12 weeks were associated with decreased OS (baseline: HR 1.06, $p<0.001 ; 6$ weeks: HR 1.07, $p=0.001$; 12 weeks: HR 1.05, $p<0.001)$. When the serial measurements of NLR measured longitudinally at baseline, 6 and 12 weeks are considered as a timedependent covariate, a Cox $\mathrm{PH}$ analysis continued to support its strongly significant association with decreased OS $(\mathrm{HR}=1.06, p<0.001)$.

NLR $>3.5$ and NLR $>5$ have both been used to define the elevated NLR group [15]. As shown in Fig. 1, there were strongly significant differences in OS between the elevated $(>3.5)$ and non-elevated $(\leq 3.5)$ NLR groups at baseline (NLR $\leq 3.5$ group median OS not reached vs. NLR $>3.5$ group median OS 11.6 months, $p=0.003$ ), at 6 weeks (median OS not reached vs 11.4 mos, $p=0.001$ ) and at 12 weeks (median OS not reached vs $11.6 \mathrm{mos}$, $p=0.001$ ). The same held true when using an NLR cutoff of 5 . The median OS in the non-elevated $(\leq 5)$ NLR group at baseline, 6 weeks, and 12 weeks were not reached; the median OS in the elevated $(>5)$ NLR group at baseline was 9.08 months, at 6 weeks was 11 months, and at 12 weeks was 11 months $(p \leq 0.009)$.

Baseline weight and baseline NLR were negatively correlated (cor $=-0.267, p=0.001$ ), and the change in weight from baseline to 12 weeks and 12 week-NLR were also inversely related (cor $=-0.371, p<0.001$ ). The 
Table 1 Demographics

\begin{tabular}{|c|c|c|}
\hline \multicolumn{3}{|c|}{ Age at Diagnosis (years) } \\
\hline & Median & 68 \\
\hline & Mean & 65 \\
\hline \multicolumn{3}{|c|}{ Gender (n, \%) } \\
\hline & Female & $58(42 \%)$ \\
\hline & Male & $81(58 \%)$ \\
\hline \multicolumn{3}{|c|}{ Ethnicity (n, \%) } \\
\hline & Caucasian & $94(67.6 \%)$ \\
\hline & African American & $35(25.2 \%)$ \\
\hline & Hispanic & $6(4.3 \%)$ \\
\hline & Asian & $3(2.2 \%)$ \\
\hline & Other & $1(0.7 \%)$ \\
\hline \multicolumn{3}{|c|}{ Smoking History At Diagnosis (n, \%) } \\
\hline & Current Smoker & $56(40.3 \%)$ \\
\hline & Former Smoker & $72(51.8 \%)$ \\
\hline & Never Smoker & $11(7.91 \%)$ \\
\hline \multicolumn{3}{|c|}{ Stage at Diagnosis (n, \%) } \\
\hline & Stage $I^{a}$ & $2(1.4 \%)$ \\
\hline & Stage $\|^{a}$ & $10(7.2 \%)$ \\
\hline & Stage III & $54(38.9 \%)$ \\
\hline & Stage IV & $72(51.8 \%)$ \\
\hline & Other & $1(0.7 \%)$ \\
\hline \multicolumn{3}{|c|}{ Histopathology (n, \%) } \\
\hline & Adenocarcinoma & $92(66.2 \%)$ \\
\hline & Squamous Cell & $42(30.2 \%)$ \\
\hline & Large Cell & $2(1.4 \%)$ \\
\hline & Other & $3(2.2 \%)$ \\
\hline \multicolumn{3}{|c|}{ Performance Status (n, \%) } \\
\hline & 0 & $44(31.6 \%)$ \\
\hline & 1 & $71(51.1 \%)$ \\
\hline & 2 & $21(15.1 \%)$ \\
\hline & 3 & $3(2.2 \%)$ \\
\hline & 4 & $0(0 \%)$ \\
\hline
\end{tabular}

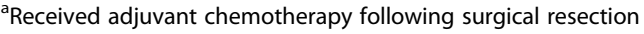

Table 2 Neutrophil-to-Lymphocyte Ratios at Baseline, 6 Weeks \& 12 Weeks

\begin{tabular}{llcl}
\hline Interval & Change in NLR $(\mathrm{n}, \%)$ & Median NLR & Range \\
\hline Baseline & - & 3.6 & $0.1898-30.910$ \\
6 Weeks & Increase $(63,45.6 \%)$ & 3.11 & $0.2703-42.11$ \\
& Decrease $(75,54.4 \%)$ & & \\
& N/A $(1)$ & & \\
12 Weeks & Increase $(67,51.9 \%)$ & 3.52 & $0.2147-42.93$ \\
& Decrease $(62,48.1 \%)$ & & \\
& N/A (10) & & \\
\hline
\end{tabular}

serial measurements of weight and NLR were found to be significantly associated in a longitudinal mixed effects regression analysis (slope $=-0.06, p<0.001$ ).

The range of weight change was $-13.17 \mathrm{~kg}$ to $+16.61 \mathrm{~kg}$ (median $-0.5 \mathrm{~kg}$, mean $-0.89 \mathrm{~kg}$ ) over 12 weeks from initiation of chemotherapy. 55 patients experienced weight gain $\geq+0.1 \mathrm{~kg}$ at 12 weeks; of that group, 41 patients exhibited weight gain at 6 weeks. Of the 55 patients who gained weight at 12 weeks, the median weight gain was $+2.41 \mathrm{~kg} .73$ patients lost weight at 12 weeks, and the median weight loss was $-3.08 \mathrm{~kg}$ (Table 3). 37 patients gained albumin at 12 weeks; of that group, 25 patients had gained albumin at 6 weeks. The median albumin gain was $+0.4 \mathrm{~g} / \mathrm{dL}$. 40 patients had a decrease in albumin at 12 weeks, with a median loss of $-0.2 \mathrm{~g} / \mathrm{dL}$. 20 patients had no change in their albumin (Table 3 ). Although there is variability amongst individual patients, there is a longitudinal inverse relationship between NLR and weight change (cor $-0.06224, p<0.001)$. There was no observed association between weight change and albumin change at 6 or 12 weeks $(p=0.265)$.

In addition, of the 96 patients who had a baseline ALI score available (Table 4), 38 (39.5\%) patients had a baseline $\mathrm{ALI}<18$ with median OS of 9.6 months compared to the 58 patients with $\mathrm{ALI} \geq 18$ with median OS not reached $(p=0.001)$. Of 93 patients who had a 6 week ALI score available, 41 (44\%) had an ALI $<18$ with median OS 11.4 months compared to the 52 patients with ALI $>18$ with median OS not reached $(p=0.03)$. Of the 84 patients who had a 12 week ALI score available, 30 (35.7\%) had an ALI $<18$ with median OS of 9 months compared to the 54 patients with $A L I \geq 18$ with median OS not reached $(p<0.001)$.

Tumor response was calculated at both 6 and 12week intervals. In total, there were 85 patients for whom a 6-week response was measurable and 95 patients for whom a 12-week response was measurable. Response data were not available for 54 patients at the 6-week time point and for 44 patients at the 12 -week time point because they had evaluable disease only based on RECIST 1.1 response criteria or a CT scan was not performed at that time point. Using a cutoff of NLR $>5$, there was a significant association between progressive disease and NLR $>5$ at 6 weeks $(p=0.02)$ and at 12 weeks $(p=0.03)$. With a cutoff of NLR $>3.5$, there was a significant association between progressive disease and NLR $>3.5$ at 6 weeks $(p=0.02)$, with a trend toward significance at 12 weeks $(p=0.06)$.

\section{Discussion}

Our understanding of the relationship between cancer cachexia, inflammation, and survival in NSCLC continues to evolve. Pretreatment ALI (BMI*Albumin/NLR) has been one attempt to bridge these three concepts, and was 

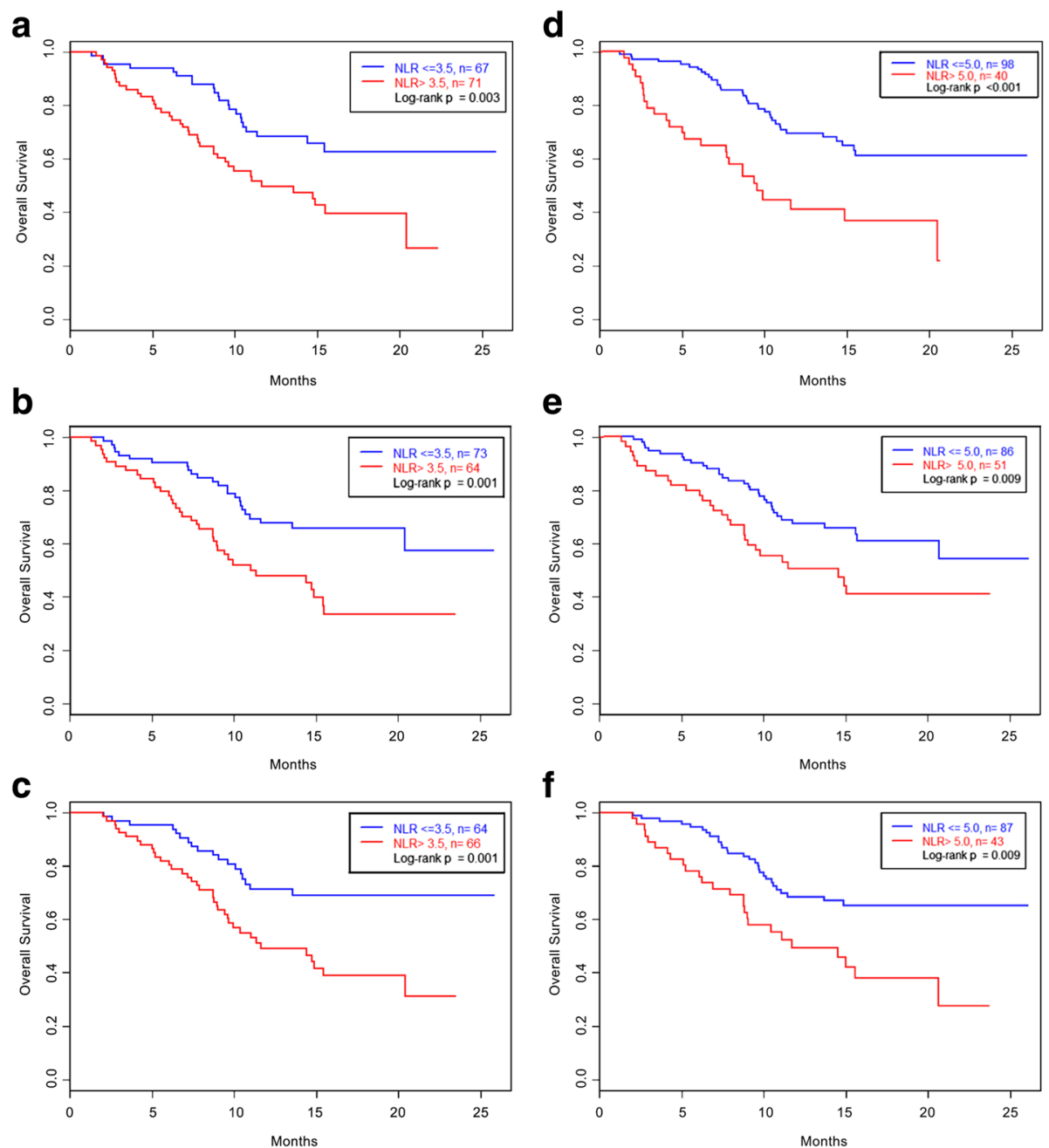

Fig. 1 Kaplan Meier Survival Plots by NLR. Kaplan Meier survival plots were generated using an NLR cutoff of 3.5 at baseline (a), at 6 weeks (b), and at 12 weeks (c) and an NLR cutoff of 5 at baseline (d), at 6 weeks (e), and at 12 weeks (f). A significant difference in overall survival was seen in all three categories, illustrating that an NLR $>3.5$ and an NLR $>5$ serve as poor prognostic factors associated with worse overall survival at diagnosis and during chemotherapy

Table 3 Changes in Weight and Albumin at $6 \& 12$ Weeks

\begin{tabular}{lcl}
\hline $\begin{array}{l}\text { Weight Change at } 6 \text { Weeks (n) } \\
\text { Increase (55) }\end{array}$ & Weight Change at 12 Weeks (n) & Median Weight Change at 12 Weeks \\
Decrease (80) & Decrease (73) & $+2.41 \mathrm{~kg}$ \\
No change (4) & No change (1) & $-3.08 \mathrm{~kg}$ \\
& N/A (10) & $0 \mathrm{~kg}$ \\
Albumin Change at 6 Weeks (n) & Albumin Change at 12 Weeks (n) & Median Albumin Change at 12 Weeks \\
Increase (36) & Increase (37) & $+0.4 \mathrm{~g} / \mathrm{dl}$ \\
$\begin{array}{l}\text { Decrease (59) } \\
\text { No Change }\end{array}$ & Decrease (40) & $-0.2 \mathrm{~g} / \mathrm{dl}$ \\
N/A (44) & No Change (20) & $0 \mathrm{~g} / \mathrm{dl}$ \\
No. Maintained Weight Gain from Weeks 6 to 12 & N/A (42) & $41 / 55(74.5 \%)$ \\
No. Maintained Albumin Gain from Weeks 6 to 12 & & $25 / 37(67.6 \%)$ \\
\hline
\end{tabular}


Table 4 Advanced Lung Cancer Inflammation Index

\begin{tabular}{llll}
\hline & Baseline $(n=97)$ & 6 Weeks $(n=94)$ & 12 Weeks $(n=84)$ \\
\hline ALI Range & $1.73-497.36$ & $0-337.16$ & $1.15-429.79$ \\
ALI Median & 21.61 & 25.02 & 25.71 \\
ALI $<18$ Median OS & 9.6 Months & 11.4 Months & 9 Months \\
ALI $\geq 18$ Median OS & Not Reached & Not Reached & Not Reached \\
$p$-value & $p=0.001$ & $p=0.03$ & $p<0.001$ \\
\hline
\end{tabular}

shown to be an independent marker of poor outcome in patients with advanced NSCLC when the ALI $<18$ compared to ALI $\geq 18$ [16]. Those patients had more sites of metastatic disease, and poorer PFS ( 2.4 vs. 5.1 months, $p<0.001)$ and OS (3.4 vs. 8.3 months, $p<0.001)$. Our findings here confirm that a pretreatment ALI $<18$ is associated with poorer OS in advanced stage NSCLC.

Tumor markers such as carcinoembryonic antigen (CEA), cancer antigen 125 (CA-125), and squamous cell carcinoma (SCC) antigen have been used to assist in diagnosis and prognosis in lung cancer but are not definitive $[17,18]$. The advantage of using the NLR alone as a longitudinal prognostic marker is in its ease of use. The ability to use a single calculation to estimate survival, derived only from a complete blood count with differential, is attractive. A recent meta-analysis demonstrated that an elevated NLR is associated with shorter progression free and overall survival in both NSCLC and SCLC, though the authors note the study was limited by bias in publication, selection, and heterogeneity [19]. Another meta-analysis found that the NLR had consistent prognostic value for overall survival in NSCLC with a cutoff value of 5 [20]. In patients receiving first-line epidermal growth factor receptor tyrosine kinase inhibitors (EGFR TKIs), the data are heterogeneous. While one study showed that an elevated NLR $\geq 3.5$ was associated with poor outcomes in EGFRmutated advanced NSCLC [15], another has shown that NLR did not affect survival in EGFR-mutated advanced NSCLC [21].

The data presented here not only shows that higher baseline NLRs are associated with inferior OS, but that progressive increases in NLR during treatment also portend a worse prognosis. Importantly, longitudinal NLRs are inversely related to both overall survival and serial body weights. Thus, the NLR has the potential for prognostication throughout a patient's treatment course. This is confirmed by the evidence that shows a significant association between progressive disease and NLR $>5$ at both 6 and 12 weeks following therapy initiation. These findings suggest that patients with higher systemic inflammation at diagnosis may have more aggressive disease and should be treated promptly and potently, while an increasing NLR during treatment may be a harbinger of disease progression and treatment failure.
Our findings are in line with evidence suggesting the role of neutrophils in modulating the cancer milieu. Neutrophils have the ability to suppress antitumor immunity, promote tumor cell proliferation, and enhance tumor cell survival, all which serve to promote tumor growth. Aging neutrophils are phagocytosed by macrophages, which have the ability to increase vascular permeability that enables tumor cell intravasation and metastasis [22]. Supporting the potential specific association between NLR and survival in NSCLC are studies in animal models with a STK11/LKB1-inactivating mutation. Inactivation of this tumor suppressor gene led to significant increases in tumor-promoting cytokines and neutrophils with T-cell suppressive effects. Interestingly, when an IL-6 antibody was introduced to the murine model, the mice experienced both a significant decrease in tumor-associated neutrophils and a significant improvement in survival compared to the control mice [23].

\section{Conclusions}

It is likely that NLR is a surrogate for ongoing inflammation, and that inflammation may be a linchpin that links tumor progression with cachexia and overall survival. While previous studies have shown the prognostic value of baseline NLR, it has been suggested that longitudinal NLRs may be more informative for individual patients [14]. The negative correlation between longitudinal NLRs, overall survival, and body weights observed in our patients suggests that simply measuring longitudinal NLRs may provide important prognostic information and may serve as a real-time indicator of disease progression and the degree of inflammation occurring in individual NSCLC patients. If this observation is confirmed in larger studies, it could have important implications for developing treatments for cancer cachexia and for designing combinations of immune modulators.

\footnotetext{
Abbreviations

ALI: Advanced lung cancer inflammation index; BMI: Body mass index; CXI: Cachexia index; EGFR: Epidermal growth factor receptor; NLR: Neutrophilto-lymphocyte ratio; NSCLC: Non-small cell lung cancer; OS: Overall survival; $\mathrm{PH}$ : Proportional hazards; RECIST: Response evaluation criteria in solid tumors; TKl: Tyrosine kinase inhibitor
}

Acknowledgements Not applicable. 


\section{Funding}

No external funding source.

\section{Availability of data and materials}

The datasets generated and/or analyzed during the current study are available in the Figshare repository, https://figshare.com/articles/ NLR_WeightGain_Deidentified_Data_csv/4604518.

\section{Authors' contributions}

BD Data collection, manuscript preparation \& revision. JM Data collection, manuscript preparation \& revision. MA Data collection, data analysis, manuscript preparation. SS Data collection, data analysis, manuscript revision. SB Data analysis, manuscript preparation \& revision. MB Manuscript preparation, manuscript revision. FE Manuscript preparation, manuscript revision. JB Data collection, manuscript preparation \& revision. PB Data collection, data analysis, manuscript preparation. MF Data collection, manuscript preparation \& revision. All authors have read and approved the final version of this manuscript.

\section{Competing interests}

The authors declare that they have no competing interests.

\section{Consent for publication}

No individual person's data is contained within the manuscript.

\section{Ethics approval and consent to participate}

This study was approved by the institutional review board at Rush University Medical Center (\#1303080), and the requirement for obtaining patient consents was waived the IRB. As such, permission to publish deidentified data was granted by said board.

\section{Author details}

${ }^{1}$ Rush University Medical Center, Chicago, IL, USA. ${ }^{2}$ University of Texas Southwestern Medical Center, Dallas, TX, USA. ${ }^{3}$ Department of Internal Medicine, 1717 W Congress Parkway, 1025 Kellogg, Chicago, IL 606012, USA. ${ }^{4}$ Division of Hematology/Oncology, 1725 W. Harrison St., Suite 809, Chicago, IL 60612, USA. ${ }^{5}$ Division of Hematology/Oncology, 5323 Harry Hines Boulevard, Dallas, TX 75390, USA. ${ }^{6}$ Department of Pathology, 1750 W. Harrison St., Suite 1415, Chicago, IL 60612, USA. ${ }^{7}$ Department of Internal Medicine, 1717 W. Congress Parkway, 10 Kellogg, Chicago, IL 60612, USA.

\section{Received: 13 September 2016 Accepted: 8 February 2017}

\section{Published online: 16 February 2017}

\section{References}

1. Fearon K, Strasser F, Anker SD, Bosaeus I, Bruera E, Fainsinger RL, et al. Definition and classification of cancer cachexia: an international consensus. Lancet Oncol. 2011;12:489-95.

2. Dewys WD, Begg C, Lavin PT, Band PR, Bennett JM, Bertino JR, et al. Prognostic effect of weight loss prior to chemotherapy in cancer patients. Eastern Cooperative Oncology Group. Am J Med. 1980;69:491-7.

3. Jatoi A, Daly BD, Hughes VA, Dallal GE, Kehayias J, Roubenoff R. Do patients with nonmetastatic non-small cell lung cancer demonstrate altered resting energy expenditure? Ann Thorac Surg. 2001;72:348-51.

4. Fearon KCH, Glass DJ, Guttridge DC. Cancer cachexia: mediators, signaling, and metabolic pathways. Cell Metab. 2012;16:153-66.

5. Gielda BT, Mehta P, Khan A, Marsh JC, Zusag TW, Warren WH, et al. Weight gain in advanced non-small-cell lung cancer patients during treatment with split-course concurrent chemoradiotherapy is associated with superior survival. Int J Radiat Oncol Biol Phys. 2011;81:985-91.

6. Patel JD, Pereira JR, Chen J, Liu J, Guba SC, John WJ, et al. Relationship between efficacy outcomes and weight gain during treatment of advanced, non-squamous, non-small-cell lung cancer patients. Ann Oncol. 2016:27:1612-9

7. Topkan E. Weight gain as a surrogate marker of longer survival in advanced non-small cell lung cancer patients. Ann Transl Med. 2016;4:381

8. Collins J, Noble S, Chester J, Coles B, Byrne A. The assessment and impact of sarcopenia in lung cancer: a systematic literature review. BMJ Open. 2014;4:e003697

9. Krenn-Pilko S, Langsenlehner U, Thurner E-M, Stojakovic T, Pichler M, Gerger A, et al. The elevated preoperative platelet-to-lymphocyte ratio predicts poor prognosis in breast cancer patients. Br J Cancer. 2014;110:2524-30.
10. Stotz M, Pichler M, Absenger G, Szkandera J, Arminger F, Schaberl-Moser R, et al. The preoperative lymphocyte to monocyte ratio predicts clinical outcome in patients with stage III colon cancer. Br J Cancer. 2014;110:435-40.

11. Hu K, Lou L, Ye J, Zhang S. Prognostic role of the neutrophil-lymphocyte ratio in renal cell carcinoma: a meta-analysis. BMJ Open. 2015;5:e006404.

12. Lee S, Oh SY, Kim SH, Lee JH, Kim MC, Kim KH, et al. Prognostic significance of neutrophil lymphocyte ratio and platelet lymphocyte ratio in advanced gastric cancer patients treated with FOLFOX chemotherapy. BMC Cancer. 2013;13:350

13. Yao Y, Yuan D, Liu H, Gu X, Song Y. Pretreatment neutrophil to lymphocyte ratio is associated with response to therapy and prognosis of advanced non-small cell lung cancer patients treated with first-line platinum-based chemotherapy. Cancer Immunol Immunother CII. 2013;62:471-9.

14. Coffelt SB, Wellenstein MD, de Visser KE. Neutrophils in cancer: neutral no more. Nat Rev Cancer. 2016:16:431-46.

15. Lin GN, Peng JW, Liu PP, Liu DY, Xiao JJ, Chen XQ. Elevated neutrophil-tolymphocyte ratio predicts poor outcome in patients with advanced nonsmall-cell lung cancer receiving first-line gefitinib or erlotinib treatment. Asian Pacific Organization for Cancer Prevention. 2014. doi: 10.1111/ajco. 12273. [Epub ahead of print].

16. Jafri SH, Shi R, Mills G. Advance lung cancer inflammation index (ALI) at diagnosis is a prognostic marker in patients with metastatic non-small cell lung cancer (NSCLC): a retrospective review. BMC Cancer. 2013;13:158.

17. Arrieta O, Villarreal-Garza C, Martínez-Barrera L, Morales M, DorantesGallareta Y, Peña-Curiel O, et al. Usefulness of serum carcinoembryonic antigen (CEA) in evaluating response to chemotherapy in patients with advanced non small-cell lung cancer: a prospective cohort study. BMC Cancer. 2013;13:254

18. Molina R, Filella X, Augé JM, Fuentes R, Bover I, Rifa J, et al. Tumor markers (CEA, CA 125, CYFRA 21-1, SCC and NSE) in patients with non-small cell lung cancer as an aid in histological diagnosis and prognosis. Tumor Biol. 2003;24:209-18

19. Zhao Q-T, Yang Y, Xu S, Zhang X-P, Wang H-E, Zhang H, et al. Prognostic role of neutrophil to lymphocyte ratio in lung cancers: a meta-analysis including 7,054 patients. Onco Targets Ther. 2015:8:2731-8.

20. Gu X-B, Tian T, Tian X-J, Zhang X-J. Prognostic significance of neutrophil-tolymphocyte ratio in non-small cell lung cancer: a meta-analysis. Sci Rep. 2015;5:12493

21. Sim SH, Beom S-H, Ahn Y-O, Keam B, Kim TM, Lee S-H, et al. Pretreatment neutrophil-lymphocyte ratio is not a significant prognostic factor in epidermal growth factor receptor-mutant non-small cell lung cancer patients treated with tyrosine kinase inhibitors. Thorac Cancer. 2016;7:161-6.

22. Engblom C, Pfirschke C, Pittet MJ. The role of myeloid cells in cancer therapies, Nat Rev Cancer. 2016:16:447-62.

23. Koyama S, Akbay EA, Li YY, Aref AR, Skoulidis F, Herter-Sprie GS, et al. STK11/ LKB1 deficiency promotes neutrophil recruitment and proinflammatory cytokine production to suppress T-cell activity in the lung tumor microenvironment. Cancer Res. 2016;76:999-1008.

\section{Submit your next manuscript to BioMed Central and we will help you at every step:}

- We accept pre-submission inquiries

- Our selector tool helps you to find the most relevant journal

- We provide round the clock customer support

- Convenient online submission

- Thorough peer review

- Inclusion in PubMed and all major indexing services

- Maximum visibility for your research

Submit your manuscript at www.biomedcentral.com/submit
Biomed Central 